FERMILAB-PUB-12-618-E

PREPARED FOR SUBMission to JHEP

\title{
A new quantity for studies of dijet azimuthal decorrelations
}

\author{
M. Wobisch, K. Chakravarthula, R. Dhullipudi, L. Sawyer, M. Tamsett \\ Department of Physics, Louisiana Tech University, \\ 600 West Arizona Ave., Ruston, USA \\ E-mail: wobisch@latech.edu, kchakrav@fnal.gov, ram.dhullipudi@cern.ch, \\ sawyer@latech.edu, tamsett@cern.ch
}

\begin{abstract}
We introduce a new measurable quantity, $R_{\Delta \phi}$, for studies of the rapidity and transverse momentum dependence of dijet azimuthal decorrelations in hadron-hadron collisions. In pQCD, $R_{\Delta \phi}$ is computed as a ratio of three-jet and dijet cross sections in which the parton distribution functions cancel to a large extent. At the leading order, $R_{\Delta \phi}$ is proportional to $\alpha_{s}$, and the transverse momentum dependence of $R_{\Delta \phi}$ can therefore be exploited to determine $\alpha_{s}$. We compute the NLO pQCD theory predictions and non-perturbative corrections for $R_{\Delta \phi}$ at the LHC and the Tevatron and investigate the corresponding uncertainties. From this, we estimate the theory uncertainties for $\alpha_{s}$ determinations based on $R_{\Delta \phi}$ at both colliders. The potential of $R_{\Delta \phi}$ measurements for tuning Monte Carlo event generators is also demonstrated.
\end{abstract}

KEYWORDS: jets, hadronic colliders, QCD 


\section{Contents}

1 Introduction 1

2 Definition and Phase Space Scenarios 3

2.1 Variables for the Rapidity and $p_{T}$ Dependence 3

2.2 Definition of $R_{\Delta \phi}$

2.3 Phase Space Scenarios for the LHC and the Tevatron 5

3 Theory Predictions and their Properties $\quad 6$

$\begin{array}{lll}3.1 & \text { NLO pQCD Predictions } & 7\end{array}$

$\begin{array}{lll}3.2 & \text { Non-perturbative Effects } & 9\end{array}$

4 Phenomenology 13

$\begin{array}{ll}\text { 4.1 Sensitivity to } \alpha_{s} & 13\end{array}$

$\begin{array}{ll}4.2 \text { Event Generator Tuning } & 15\end{array}$

$\begin{array}{lll}5 & \text { Summary } & 16\end{array}$

\section{Introduction}

Theory predictions for inclusive jet and dijet cross sections in hadron-hadron collisions at fixed order in perturbative Quantum Chromodynamics (pQCD) are currently available at next-to-leading order (NLO) in the strong coupling constant $\alpha_{s}$. Using precise experimental data, these predictions have been well tested and applied in determining the parton distribution functions (PDFs) of the proton, and $\alpha_{s}$ [1]. Direct tests of pQCD at higher orders require measurements of quantities probing multi-jet final states with three or more jets. Quantities in which a cross section for the production of three or more jets is normalized by a dijet cross section (or an inclusive jet cross section) are ideal for $\alpha_{s}$ determinations. These quantities are still sensitive to the degrees of freedom in the multi-jet final state and, in pQCD, proportional to (at least) $\mathcal{O}\left(\alpha_{s}\right)$, while the PDF sensitivity exhibited by a typical multi-jet cross section [2] can be strongly reduced. Examples of such quantities are the ratio of the inclusive three jet and dijet cross sections, $R_{3 / 2}$ [3-5], and the average number of neighboring jets, $R_{\Delta R}$, which has recently been proposed, measured, and used to determine $\alpha_{s}[6]$.

A third related quantity is the dijet azimuthal decorrelation, which studies the relative angle in the azimuthal plane between the two jets with the highest transverse momentum $\left(p_{T}\right) \Delta \phi_{\text {dijet }}=\left|\phi_{\text {jet1 }}-\phi_{\text {jet2 } 2}\right|$. In calculations at $\mathcal{O}\left(\alpha_{s}^{2}\right)$, dijet events have exactly two jets with equal $p_{T}$, and their azimuthal angles are correlated such that $\Delta \phi_{\text {dijet }}=\pi$. Deviations from $\Delta \phi_{\text {dijet }}=\pi$ (hereafter referred to as "azimuthal decorrelations") are caused by additional 

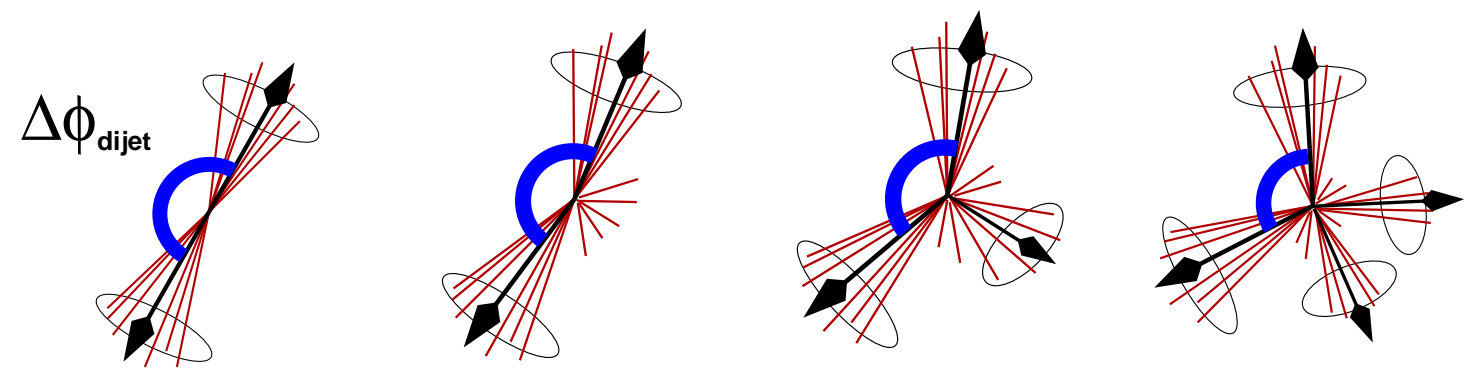

Figure 1. A sketch of the angle $\Delta \phi_{\text {dijet }}$ in the azimuthal plane, in dijet events for different amounts of additional radiation outside the dijet system.

radiation which is not clustered into the two highest $p_{T}$ jets. Additional radiation with small $p_{T}$ reduces $\Delta \phi_{\text {dijet }}$ by a small amount, while high- $p_{T}$ radiation can reduce $\Delta \phi_{\text {dijet }}$ significantly thereby leading to larger azimuthal decorrelations as illustrated in figure 1 . Due to kinematic constraints, three-jet final states are restricted to $\Delta \phi_{\text {dijet }}>2 \pi / 3$, while the phase space of $\Delta \phi_{\text {dijet }}<2 \pi / 3$ is only accessible in final states with at least four jets.

The $\mathrm{D} \varnothing$ collaboration has introduced the quantity $\left(1 / \sigma_{\text {dijet }}\right) \cdot d \sigma_{\text {dijet }} / d \Delta \phi_{\text {dijet }}$, which is the dijet cross section differentially in $\Delta \phi_{\text {dijet }}$, normalized by the inclusive dijet cross section $\sigma_{\text {dijet }}$ (in the same kinematic range and integrated over $\Delta \phi_{\text {dijet }}$ ) [7]. This quantity was measured in $p \bar{p}$ collisions at $\sqrt{s}=1.96 \mathrm{TeV}$, for different $p_{T}^{\max }$ requirements, where $p_{T}^{\max }$ is the highest jet $p_{T}$ in the event, and for a fixed $p_{T}$ requirement for the second leading $p_{T}$ jet. For this quantity, the range from small to large azimuthal decorrelations can be used to study the transition from soft to hard higher-order pQCD processes and the measurement results placed strong constraints on Monte Carlo parameters [8]. The same analysis strategy was later employed by the CMS and ATLAS collaborations using $p p$ collision data at $\sqrt{s}=$ $7 \mathrm{TeV}$, thus accessing larger $p_{T}^{\max }[9,10]$. The common approach focuses on the $\Delta \phi_{\text {dijet }}$ dependence; the $p_{T}$ dependence is not easily visible in these presentations. Furthermore, in pQCD, dijet azimuthal decorrelations are predicted to depend not only on $p_{T}$, but also on the rapidities of the two leading $p_{T}$ jets. The measurements by the DØ, CMS, and ATLAS collaborations, however, did not explore the rapidity dependence.

In this article, we propose a new quantity $R_{\Delta \phi}$ for studying dijet azimuthal decorrelations with emphasis on the rapidity and the $p_{T}$ dependence. ${ }^{1}$ The former aspect will allow us to perform novel tests of the pQCD predictions, while the latter can be exploited for determinations of $\alpha_{s}$ and its running. The article is structured as follows: In section 2 we motivate the variables used to study the rapidity and $p_{T}$ dependencies, and we give the definition of $R_{\Delta \phi}$. In addition, we propose realistic scenarios for phase space regions in which $R_{\Delta \phi}$ can be measured by the LHC and the Tevatron experiments. Theory predictions for these scenarios are presented in section 3, including perturbative and non-perturbative contributions. The possible impact of $R_{\Delta \phi}$ measurements on determinations of $\alpha_{s}$ and on Monte Carlo tuning is discussed in section 4.

\footnotetext{
${ }^{1}$ Some initial studies for experimental measurements of $R_{\Delta \phi}$ have been made in references [11, 12].
} 


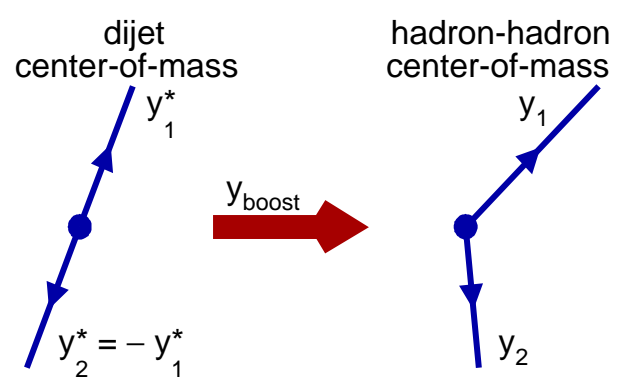

Figure 2. Jet rapidity variables in the transverse plane in the dijet center-of-mass frame and in the hadron-hadron center-of-mass frame.

\section{Definition and Phase Space Scenarios}

The quantities $R_{3 / 2}[3-5]$ and $R_{\Delta R}[6]$ are defined as ratios of multi-jet cross sections. These ratios can be interpreted as the conditional probability that an event with two high- $p_{T}$ jets also contains a third jet $\left(R_{3 / 2}\right)$ and as the average number of neighboring jets for a given jet $\left(R_{\Delta R}\right)$. We propose to study dijet azimuthal decorrelations, using a quantity with a similar intuitive interpretation. For this purpose we introduce the quantity $R_{\Delta \phi}$. Before we define $R_{\Delta \phi}$, we motivate the variables used to study the rapidity and $p_{T}$ dependencies. In addition, we propose realistic scenarios for measurements of $R_{\Delta \phi}$ at the LHC and the Tevatron.

\subsection{Variables for the Rapidity and $p_{T}$ Dependence}

The Rapidity Variable One of the main goals for the new quantity $R_{\Delta \phi}$ is to measure the rapidity dependence of dijet azimuthal decorrelations. The previous analyses $[7,9,10]$ applied rapidity requirements for both jets in the hadron-hadron center-of-mass frame (i.e. the lab frame). In general, this frame is, however, longitudinally boosted with respect to the center-of-mass frame of the hard subprocess (corresponding to $y_{\text {boost }}$ ), as shown in figure 2. In the approximation of $2 \rightarrow 2$ processes, the rapidities $y_{1}^{*}$ and $y_{2}^{*}$ (in the dijet center-of-mass frame) have the same magnitude $\left(y^{*} \equiv\left|y_{1}^{*}\right|=\left|y_{2}^{*}\right|\right)$, and are related to the rapidities $y_{1}$ and $y_{2}$ (in the hadron-hadron center-of-mass frame) by

$$
y_{1}=y_{1}^{*}+y_{\text {boost }} \quad \text { and } \quad y_{2}=y_{2}^{*}+y_{\text {boost }} .
$$

We propose to measure the rapidity dependence of $R_{\Delta \phi}$ as a function of the variable $y^{*}$ for a fixed requirement for the variable $y_{\text {boost. }}$ Both variables are given by

$$
y_{\text {boost }}=\left(y_{1}+y_{2}\right) / 2 \quad \text { and } \quad y^{*}=\left|y_{1}-y_{2}\right| / 2,
$$

where $y_{1}$ and $y_{2}$ are the respective rapidities of the two leading $p_{T}$ jets in the event.

The $\boldsymbol{p}_{\boldsymbol{T}}$ Variable In the leading logarithmic approximation, an $n$-parton final state can be regarded as emerging from a two-parton final state through successive branching, as displayed in figure 3 for three- and four-parton final states. In this picture, a quantity such 


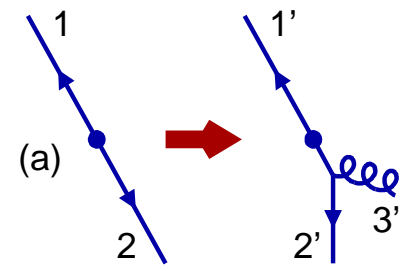

$\mathrm{p}_{\mathrm{T} 1}=\mathrm{p}_{\mathrm{T} 1}$,

$\left(\mathrm{p}_{\mathrm{T} 1}+\mathrm{p}_{\mathrm{T} 2}\right)<\left(\mathrm{p}_{\mathrm{T} 1},+\mathrm{p}_{\mathrm{T} 2^{\prime}}\right)$

$\mathrm{H}_{\mathrm{T}}=\Sigma_{\mathrm{i}}\left(\mathrm{p}_{\mathrm{Ti}_{\mathrm{i}}}\right) \approx \Sigma_{\mathrm{i}^{\prime}}\left(\mathrm{p}_{\mathrm{Ti}^{\prime}}\right)=\mathrm{H}_{\mathrm{T}}^{\prime}$

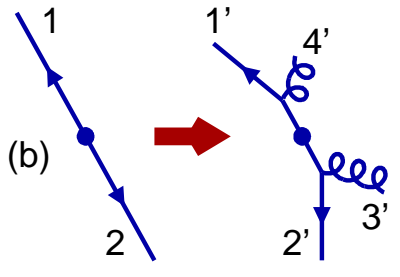

$\mathrm{p}_{\mathrm{T} 1}<\mathrm{p}_{\mathrm{T} 1}$,

$\left(\mathrm{p}_{\mathrm{T} 1}+\mathrm{p}_{\mathrm{T} 2}\right)<\left(\mathrm{p}_{\mathrm{T} 1},+\mathrm{p}_{\mathrm{T} 2^{\prime}}\right)$

$\mathrm{H}_{\mathrm{T}}=\Sigma_{\mathrm{i}}\left(\mathrm{p}_{\mathrm{Ti}}\right) \approx \Sigma_{\mathrm{i}^{\prime}}\left(\mathrm{p}_{\mathrm{Ti}^{\prime}}\right)=\mathrm{H}_{\mathrm{T}}^{\prime}$

Figure 3. Sketches of the azimuthal plane in which a three-parton (a) and a four-parton final state (b) emerge from a two-parton final state through parton-branching, and the relations of different variables before and after the branching. The variable $H_{T}$ is approximately preserved in these processes.

as $R_{3 / 2}$ may be interpreted as the branching probability from two to three final state partons (for partons which have a sufficient angular separation to be resolved as individual jets, according to the jet definition). However, this interpretation only holds if the quantity is binned in a " $p_{T}$-type" variable that does not change its value before and after the branching of the third parton. Examples of such variables are the leading jet $p_{T}$, or, approximately, $H_{T}$ (defined as the scalar $p_{T}$ sum over all jets in an event). A counter example is the variable $H_{T}^{(2)}=\left(p_{T 1}+p_{T 2}\right)$, defined as the scalar $p_{T}$ sum of the two leading jets, which is reduced after the branching displayed in figure 3 (a). For a quantity like the ratio of inclusive four-jet and dijet cross sections, $R_{4 / 2}$, the leading jet $p_{T}$ could also be reduced, e.g. by a branching as displayed in figure 3 (b).

In the measurement of dijet azimuthal decorrelations, the angle $\Delta \phi_{\text {dijet }}$ is affected not only by the third jet but by all additional radiation in the event. Therefore variables like $p_{T}^{\max }$ or $H_{T}^{(2)}$ are not suited if we want to interpret the ratio $R_{\Delta \phi}$ as the probability for parton branching. Only the value of $H_{T}$ is approximately conserved after the branching processes. With an ideal detector and in a clean environment, one might want to define $H_{T}$ as the scalar $p_{T}$ sum of all jets in the event, without any $p_{T}$ or $y$ requirements. In practice, one has a limited detector $y$ acceptance, a limited knowledge of the detector response for low $p_{T}$ jets, plus contributions from the underlying event. Therefore it is advisable to restrict the $p_{T}$ sum in the $H_{T}$ definition to jets which are well measured, and for which non-perturbative contributions are small, by requiring $p_{T i}>p_{T \text { min }}$. A limited detector $y$ acceptance can be taken into account by requiring that the jets are contained inside this acceptance region. Since we study the rapidity dependence based on $y^{*}$ which is longitudinally boost invariant, we would like to preserve this property also for the $H_{T}$ definition. Therefore the jet selection is not based on the absolute jet rapidities $\left|y_{i}\right|$ in the lab frame, but on the longitudinally boost invariant quantity $\left|y_{i}-y_{\text {boost }}\right|$, and $H_{T}$ is computed as

$$
H_{T}=\sum_{i \in C} p_{T i},
$$


based on all jets in the set $C$ which is defined as

$$
C=\left\{i \mid 1 \leq i \leq n_{\text {jet }} ; \text { and } p_{T i}>p_{T \min } ; \text { and }\left|y_{i}-y_{\text {boost }}\right|<y_{\max }^{*}\right\} .
$$

In this definition, $n_{\text {jet }}$ is the total number of jets in the event, and $p_{T \min }$ and $y_{\max }^{*}$ are parameters which can be chosen according to the experimental environment. The value of $y_{\max }^{*}$ should be chosen at least as large as the maximum accessible $y^{*}$ for two leading $p_{T}$ jets, to ensure that these are always members of the set $C$, and therefore included in the $H_{T}$ sum.

\subsection{Definition of $R_{\Delta \phi}$}

With the criteria above, we propose to study dijet azimuthal decorrelations using the new quantity $R_{\Delta \phi}$, which represents the fraction of all inclusive dijet events for which the two leading $p_{T}$ jets have a decorrelation of $\Delta \phi_{\text {dijet }}<\Delta \phi_{\max }$. It is defined as

$$
R_{\Delta \phi}\left(H_{T}, y^{*}, \Delta \phi_{\max }\right)=\frac{\frac{d^{2} \sigma_{\text {dijet }}\left(\Delta \phi_{\text {dijet }}<\Delta \phi_{\max }\right)}{d H_{T} d y^{*}}}{\frac{\left.d^{2} \sigma_{\text {dijet }} \text { (inclusive }\right)}{d H_{T} d y^{*}}} .
$$

The denominator, $d^{2} \sigma_{\text {dijet }}$ (inclusive) $/\left(d H_{T} d y^{*}\right)$, is the cross section for the production of two or more jets, with $p_{T}>p_{T \min }$, and $y_{\text {boost }}<y_{\text {boost }}^{\max }$, double differentially in the variables $y^{*}$ and $H_{T}$. The numerator, $d^{2} \sigma_{\text {dijet }}\left(\Delta \phi_{\text {dijet }}<\Delta \phi_{\max }\right) /\left(d H_{T} d y^{*}\right)$, is a subset of the denominator with the additional requirement that the two leading $p_{T}$ jets have $\Delta \phi_{\text {dijet }}<\Delta \phi_{\text {max }}$. The quantity $R_{\Delta \phi}$ is measured as a function of the parameter $\Delta \phi_{\max }$, and in bins of $y^{*}$ and $H_{T}$, and therefore expressed as $R_{\Delta \phi}\left(H_{T}, y^{*}, \Delta \phi_{\max }\right)$.

It may be convenient to introduce an additional requirement of an $H_{T}$-dependent lower limit on the leading jet $p_{T}$ as $p_{T 1}>f \cdot H_{T}$, in both the numerator and the denominator. This requirement (which cuts the tail of low leading jet $p_{T}$ ) is necessary in the experiment if events are triggered by inclusive single jet triggers. The value of $f$ should not be too large, so as to not restrict the multi-jet phase space too strongly. We recommend to set $f$ not larger than $f=1 / 3$, so that the phase space for $2 \rightarrow 2$ and $2 \rightarrow 3$ processes is not affected.

\subsection{Phase Space Scenarios for the LHC and the Tevatron}

To produce specific theory predictions, we propose two scenarios of phase space regions in which $R_{\Delta \phi}$ can be measured at the LHC and the Tevatron. While making realistic choices that take into account current practices by the experiments, we try to keep the two scenarios as similar as possible, so that the results can be used to study the $\sqrt{s}$ dependence of $R_{\Delta \phi}$.

LHC Scenario We assume the running conditions of 2012, where the LHC was producing $p p$ collisions at $\sqrt{s}=8 \mathrm{TeV}$. Following the choices by the ATLAS and CMS experiments, jets are defined using the anti- $\mathrm{k}_{t}$ jet algorithm [13], here with a jet radius of $R=0.6$ (in the $y-\phi$ plane), which is within the range of $0.4-0.7$ that is used by ATLAS and CMS. The parameters in the $R_{\Delta \phi}$ definition are set to $y_{\max }^{*}=2.0, y_{\text {boost }}^{\max }=0.5$, and $p_{T \min }=100 \mathrm{GeV}$. The additional $H_{T}$-dependent requirement on the leading jet $p_{T}$ is $p_{T 1} / H_{T}>1 / 3$. The 


\begin{tabular}{|c|c|c|}
\hline & LHC & Tevatron \\
& $p p$ at $\sqrt{s}=8 \mathrm{TeV}$ & $p \bar{p}$ at $\sqrt{s}=1.96 \mathrm{TeV}$ \\
\hline jet algorithm & anti- $\mathrm{k}_{t}, R=0.6$ & Run II cone, $R_{\text {cone }}=0.7$ \\
$p_{T \min }\left(x_{T \min }\right)$ & $100 \mathrm{GeV}(0.0250)$ & $30 \mathrm{GeV}(\approx 0.0306)$ \\
$y_{\text {boost }}^{\max }$ & 0.5 & 0.5 \\
$y_{\max }^{*}$ & 2.0 & 2.0 \\
$p_{T 1} / H_{T}$ & $>1 / 3$ & $>1 / 3$ \\
$\Delta \phi_{\max }$ & $7 \pi / 8,5 \pi / 6,3 \pi / 4$ & $7 \pi / 8,5 \pi / 6,3 \pi / 4$ \\
$y^{*}$ ranges & $0.0-0.5,0.5-1.0,1.0-2.0$ & $0.0-0.5,0.5-1.0,1.0-2.0$ \\
$H_{T}$ range & $750-4000 \mathrm{GeV}$ & $180-900 \mathrm{GeV}$ \\
\hline
\end{tabular}

Table 1. Summary of the phase space definitions for the LHC and the Tevatron scenarios.

$y_{\max }^{*}$ and $y_{\text {boost }}^{\max }$ requirements ensure that the two leading $p_{T}$ jets and all other jets entering the $H_{T}$ sum are well-contained in the detector, within $|y|<2.5$. The $p_{T \text { min }}$ requirement ensures that all jets are well measured in the experiment, and that pileup contributions and non-perturbative corrections are small. For studies of the $\sqrt{s}$ dependence of $R_{\Delta \phi}$, we note that the $p_{T \text { min }}$ requirement translates to a requirement for the scaling variable $x_{T}=2 p_{T} / \sqrt{s}$ of $x_{T \min }=0.025$. The parameter $\Delta \phi_{\max }$ is set to $7 \pi / 8,5 \pi / 6$, or $3 \pi / 4$, and the $y^{*}$ regions are chosen as $0<y^{*}<0.5,0.5<y^{*}<1$, and $1<y^{*}<2$. The $H_{T}$ dependence is studied over the range $750<H_{T}<4000 \mathrm{GeV}$.

Tevatron Scenario In Run II, the Tevatron collided protons and anti-protons at $\sqrt{s}=$ $1.96 \mathrm{TeV}$. For the majority of the jet results, the $\mathrm{CDF}$ and $\mathrm{D} \varnothing$ experiments use iterative seed-based cone algorithms with a cone of radius $R_{\text {cone }}=0.7$ in $y$ and $\phi$. For these studies, we apply the Run II midpoint cone jet algorithm [14] that is used by DØ. We use the same values for the parameters $y_{\max }^{*}, y_{\text {boost }}^{\max }$, and $\Delta \phi_{\max }$, the identical $y^{*}$ regions, and the same $p_{T 1} / H_{T}$ requirement as in the LHC scenario. The only differences are the value of the $p_{T \text { min }}$ requirement, which is set to $p_{T \text { min }}=30 \mathrm{GeV}$, and the $H_{T}$ range of $180-900 \mathrm{GeV}$. The $p_{T \text { min }}$ requirement translates to a requirement for the scaling variable of $x_{T \min } \approx 0.0306$, which is slightly higher than the corresponding requirement in the LHC scenario (of $x_{T \min }=0.025$ ). However, we use this value because it corresponds to the lower $p_{T}$ requirements used in recent multi-jet measurements at the Tevatron $[5,6]$

The parameters, defining the phase space for the LHC and the Tevatron scenarios are summarized in table 1.

\section{Theory Predictions and their Properties}

In this section, we compute the perturbative and the non-perturbative contributions for $R_{\Delta \phi}$ and investigate their properties. We compare the predictions for the LHC and the Tevatron scenarios, and investigate the differences due to the differences in $\sqrt{s}$, and due to the slightly different phase space requirements.

All theory results have been obtained using the implementations of the anti- $\mathrm{k}_{t}$ and the DØ Run II cone jet algorithms in FASTJET [15, 16]. 

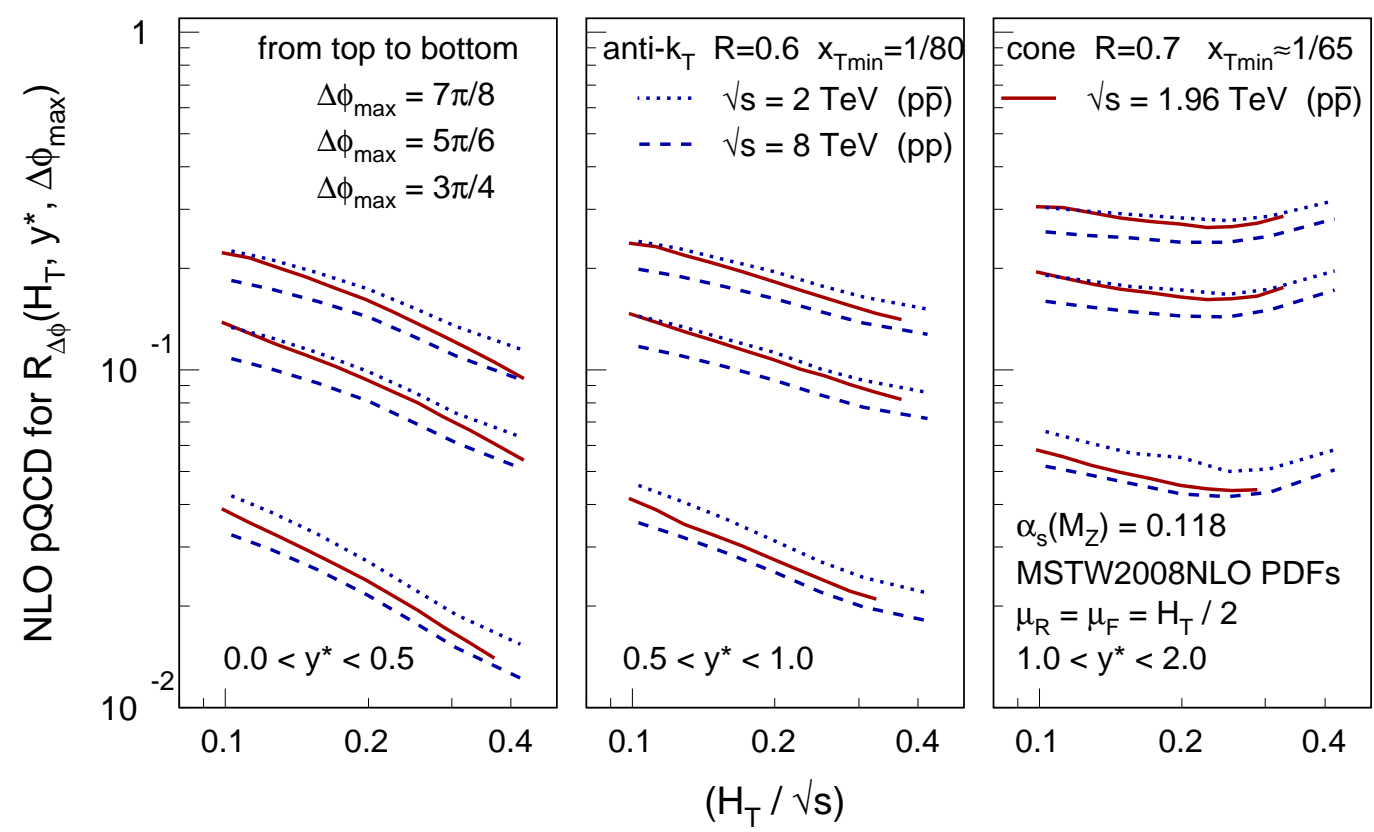

Figure 4. The NLO pQCD predictions for $R_{\Delta \phi}$ as a function of $\left(H_{T} / \sqrt{s}\right)$, and in regions of $y^{*}$ (columns) and for different $\Delta \phi_{\max }$ requirements. The results are shown for the LHC and the Tevatron scenarios, and for a LHC-like scenario in which the LHC scenario is scaled to $\sqrt{s}=2 \mathrm{TeV}$ and modified to $p \bar{p}$ collisions.

\subsection{NLO pQCD Predictions}

The NLO (LO) pQCD predictions for $R_{\Delta \phi}$ are computed by taking the ratios of the NLO (LO) pQCD predictions for the cross sections in the numerator and the denominator in eq. (2.5). The denominator is the inclusive dijet cross section for which the NLO (LO) prediction is computed at $\mathcal{O}\left(\alpha_{s}^{3}\right)\left(\mathcal{O}\left(\alpha_{s}^{2}\right)\right)$. Due to the additional requirement of $\Delta \phi_{\text {dijet }}<\Delta \phi_{\max }$, the numerator receives only contributions from final states with three or more jets. Therefore, the numerator is a three-jet cross section for which the NLO (LO) prediction is computed at $\mathcal{O}\left(\alpha_{s}^{4}\right)\left(\mathcal{O}\left(\alpha_{s}^{3}\right)\right)$. All NLO and LO pQCD results are computed using NLOJET $++[17,18]$, interfaced to FASTNLO [19]. The calculations are made in the $\overline{\mathrm{MS}}$ scheme [20] for five active quark flavors, and using the next-to-leading logarithmic (twoloop) approximation of the renormalization group equation. The value of $\alpha_{s}\left(M_{Z}\right)=0.118$ is used consistently in the matrix elements and in the MSTW2008NLO PDF sets [21]. The central choice $\mu_{0}$ for the renormalization and factorization scales is $\mu_{R}=\mu_{F}=\mu_{0}=H_{T} / 2 .^{2}$

The results of the NLO calculations for the LHC and the Tevatron scenarios are displayed in figure 4 , where $R_{\Delta \phi}$ is shown as a function of $\left(H_{T} / \sqrt{s}\right)$ in different regions of $y^{*}$ and for different $\Delta \phi_{\max }$ requirements. In different regions of $H_{T}$ and $y^{*}$, and for different choices of $\Delta \phi_{\max }, R_{\Delta \phi}$ has values in the range 0.012-0.32. In most phase space regions, $R_{\Delta \phi}$ decreases with increasing $H_{T}$, except at $1<y^{*}<2$ where $R_{\Delta \phi}$ increases again at high

\footnotetext{
${ }^{2}$ At LO, this choice coincides with the common choices of $\mu_{R, F}=p_{T}$ for inclusive jet production and $\mu_{R, F}=\left(p_{T 1}+p_{T 2}\right) / 2$ for dijet production.
} 
$H_{T}$. At fixed $H_{T}, R_{\Delta \phi}$ increases with increasing $y^{*}$. The fact that $R_{\Delta \phi}$ decreases with decreasing $\Delta \phi_{\max }$ is a trivial phase space effect, since a stronger $\Delta \phi_{\text {dijet }}$ requirement leads to a smaller cross section in the numerator.

For a fixed $x_{T \text { min }}$ requirement and at fixed $\left(H_{T} / \sqrt{s}\right)$, the $\sqrt{s}$ dependence of the perturbative results for $R_{\Delta \phi}$ is only introduced through the evolution of $\alpha_{s}$ and the PDFs with the scales $\mu_{R}$ and $\mu_{F}$. In figure 4 , the $\sqrt{s}$ dependence of $R_{\Delta \phi}$ cannot directly be judged based on the comparison of the LHC and the Tevatron scenarios, as the two differ in the $x_{T \text { min }}$ requirement and in the jet algorithm. The following study is made to separate the latter effects from the genuine $\sqrt{s}$ dependence of $R_{\Delta \phi}$. Using the flexibility provided by FASTNLO, we use the FASTNLO coefficient tables for the LHC scenario (for $p p$ collisions at $\sqrt{s}=8 \mathrm{TeV}$ ) to compute the corresponding predictions for $p \bar{p}$ collisions at the same $\sqrt{s}$. The results for the latter (not shown in figure 4) agree with those for the LHC scenario better than $0.8 \%$ for $H_{T}<2 \mathrm{TeV}$ and always better than $3.2 \%$ in the phase space studied, meaning that $R_{\Delta \phi}$ is insensitive to the difference between $p p$ and $p \bar{p}$ initial states. Then we use the FASTNLO results for the LHC scenario to compute the corresponding predictions for a LHC-like scenario (i.e. using the same jet algorithm and the same $x_{T \text { min }}$ requirement) for $p \bar{p}$ collisions at $\sqrt{s}=2 \mathrm{TeV}$. These predictions are shown in figure 4 as the dotted line. The $R_{\Delta \phi}$ results at $\sqrt{s}=2 \mathrm{TeV}$ are $10-20 \%$ higher than those at $8 \mathrm{TeV}$. This $\sqrt{s}$ dependence is consistent with the running of $\alpha_{s}$ over a factor of four in energy. ${ }^{3}$ For $\Delta \phi_{\max }=7 \pi / 8$ and $5 \pi / 6$, the results for the LHC-like scenario agree within $5 \%$ with those for the Tevatron scenario. Only for $\Delta \phi_{\max }=3 \pi / 4$ the differences become larger (slightly more than $10 \%$ at $y^{*}>1$ ). From this we conclude that, even with different jet algorithms and slightly different $x_{T \text { min }}$ requirements, a comparison of Tevatron and LHC data is probing the $\sqrt{s}$ dependence of $R_{\Delta \phi}$ and testing the corresponding theory predictions.

In the following, we investigate the NLO $k$-factors and the scale dependence as indicators for the stability of the perturbative expansion, and we study the PDF uncertainties for $R_{\Delta \phi}$. The NLO $k$-factors are computed as the ratio of the NLO and the LO predictions, $k=R_{\Delta \phi}^{\mathrm{NLO}} / R_{\Delta \phi}^{\mathrm{LO}}$. The values of the $k$-factors are displayed in figure 5 as a function of $\left(H_{T} / \sqrt{s}\right)$, for the LHC and the Tevatron scenarios. For $\Delta \phi_{\max }=7 \pi / 8$ and $5 \pi / 6$, the $k$ factors for the LHC and the Tevatron are always close to unity; they decrease slightly with increasing $y^{*}$ and are almost independent of $H_{T}$. Due to kinematic constraints, the region of $\Delta \phi_{\text {dijet }}<2 \pi / 3$ is only accessible in four-jet final states. For this reason, the kinematic region of $\Delta \phi_{\max }=3 \pi / 4$ also receives large contributions from four-jet production which are only modeled at LO by the $\mathcal{O}\left(\alpha_{s}^{4}\right)$ calculation for the numerator of $R_{\Delta \phi}$. This is reflected in the large NLO $k$-factors for $\Delta \phi_{\max }=3 \pi / 4$ which are as large as $k=1.5$ at lower $H_{T}$.

The uncertainties due to the scale dependence are computed from the relative variations of the $R_{\Delta \phi}$ results when $\mu_{R}$ and $\mu_{F}$ are varied independently around $\mu_{0}=H_{T} / 2$ between $\mu_{0} / 2$ and $2 \mu_{0}$ but never exceeding $0.5 \leq \mu_{R} / \mu_{F} \leq 2.0$. These uncertainties are displayed in figure 6 for the LHC and Tevatron scenarios. For the LHC (Tevatron) scenario, these uncertainties are typically $5 \%(7 \%)$ for $\Delta \phi_{\max }=7 \pi / 8$ and $5 \pi / 6$, and up to $19 \%(17 \%)$

\footnotetext{
${ }^{3}$ The PDFs approximately cancel in the ratio $R_{\Delta \phi}$, so the $\mu_{F}$ dependence of the PDFs does not have a significant impact on the $\sqrt{s}$ dependence of $R_{\Delta \phi}$.
} 


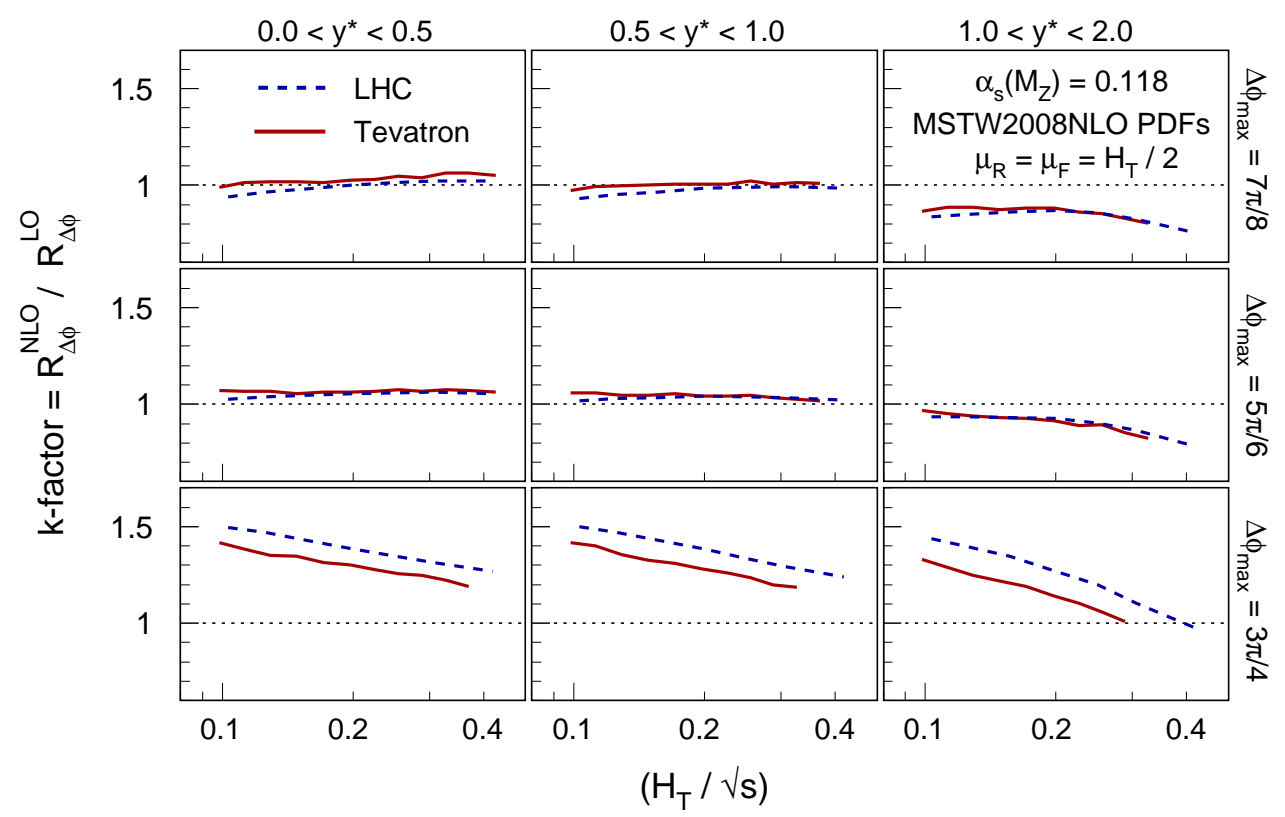

Figure 5. The NLO $k$-factors for $R_{\Delta \phi}$ as function of $\left(H_{T} / \sqrt{s}\right)$, in different regions of $y^{*}$ (columns) and for different $\Delta \phi_{\max }$ (rows), for the LHC and the Tevatron scenarios.

for $\Delta \phi_{\max }=3 \pi / 4$. The latter is directly related to the large NLO $k$-factors in this region. The uncertainties for $\Delta \phi_{\max }=7 \pi / 8$ and $5 \pi / 6$ are slightly smaller as compared to the uncertainties for other ratios of three-jet and dijet cross sections like $R_{3 / 2}$ and $R_{\Delta R}$ as recently measured at the LHC [4] and the Tevatron $[5,6]$.

The PDF uncertainties are computed for the MSTW2008NLO PDFs using the up and down variations of the 20 orthogonal PDF uncertainty eigenvectors, corresponding to the $68 \%$ C.L. The MSTW2008NLO PDF uncertainties for the LHC and Tevatron scenarios are shown in figure 6 and they are always below 1\%. Also displayed in figure 6 are the NLO pQCD predictions obtained for CT10 [22] and NNPDFv2.1 [23] PDFs. Those results are larger by up to $5 \%$ as compared to the results for MSTW2008NLO PDFs. The largest deviations occur at smallest $\Delta \phi_{\max }$.

From these studies we conclude that theory predictions are most reliable (as indicated by a small scale dependence and $k$-factors which are close to unity) in the kinematic regions of $y^{*}<1$ and for $\Delta \phi_{\max }=7 \pi / 8$ and $5 \pi / 6$.

\subsection{Non-perturbative Effects}

In these studies, we consider non-perturbative effects due to the underlying event and due to hadronization corrections. Both corrections are estimated using the models implemented in the event generators HERWIG 6.520 [24, 25] and PYTHIA 6.426 [26, 27]. The HERWIG results are obtained using default settings and the PYTHIA results are obtained for four different popular tunes. These are tunes DW [28] and A [29], which use a $Q^{2}$ ordered parton shower and an older model for the underlying event, and the tunes AMBT1 [30] and S Global [31], which use a $p_{T}$ ordered parton shower and the new model for the underlying event [32, 33]. 

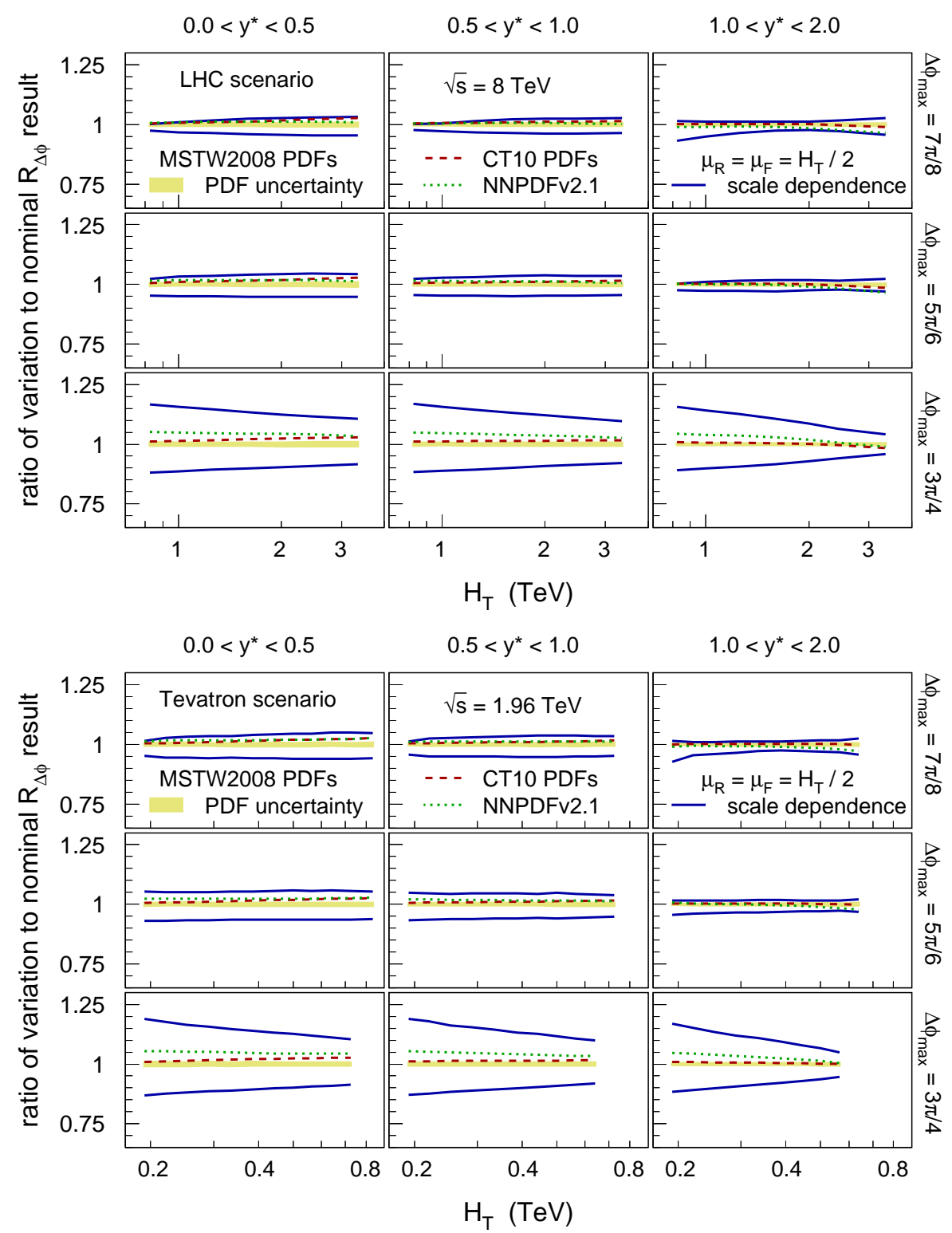

Figure 6. The renormalization and factorization scale dependence and the PDF uncertainties for the MSTW2008NLO PDFs for the NLO pQCD predictions for the LHC and Tevatron scenarios.

The non-perturbative corrections are obtained from the results of three calculations in which $R_{\Delta \phi}$ is computed

(1) at the parton level (the partons after the parton shower) with no underlying event,

(2) at the particle level (using all stable particles) with no underlying event, and

(3) at the particle level (using all stable particles) with underlying event.

The total non-perturbative correction $c_{\text {npert }}$ is defined as the product of the hadronization correction $c_{\text {hadr }}$ and the underlying event correction $c_{\text {ue }}$ which are each given by ratios of 

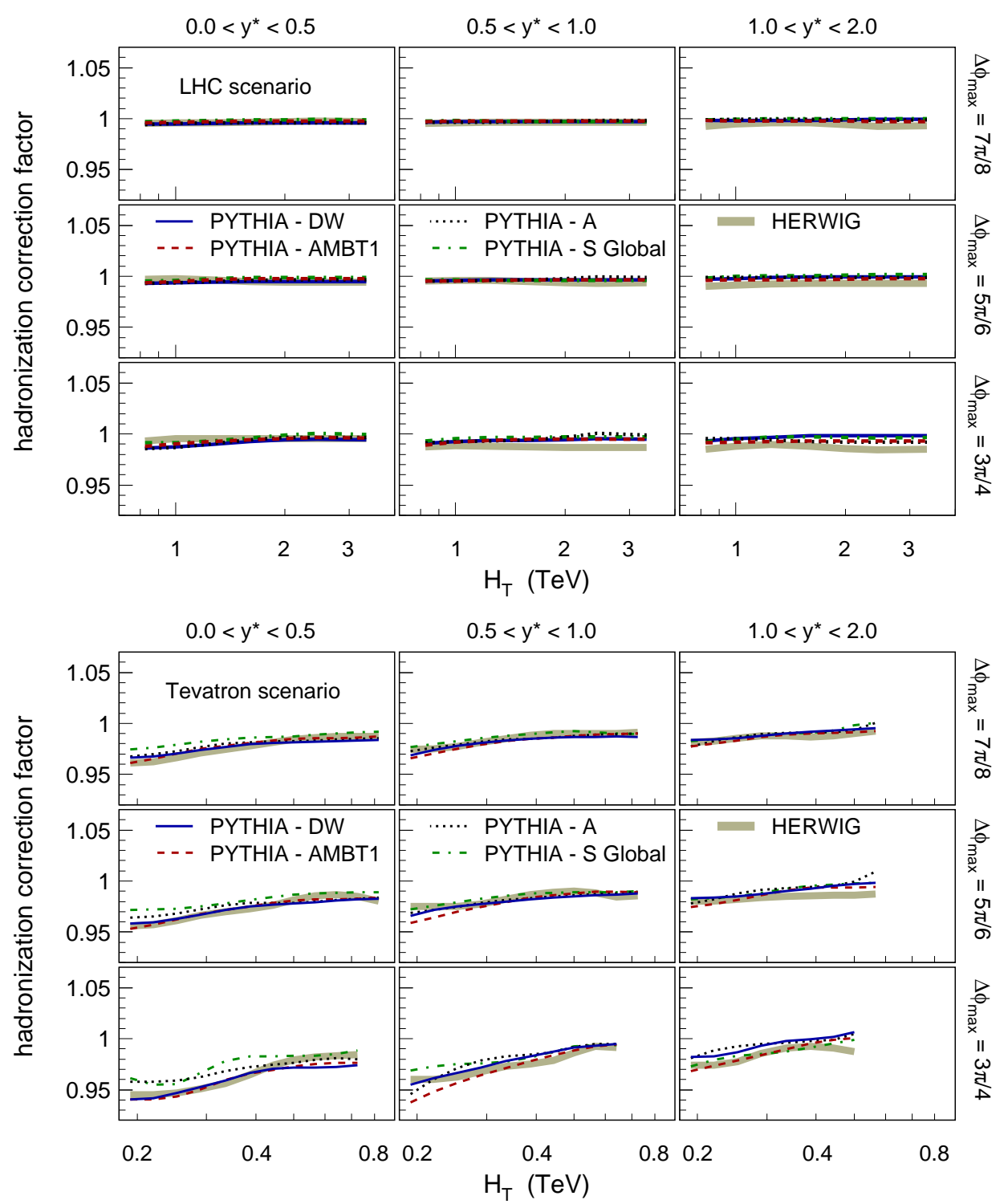

Figure 7. The hadronization corrections for $R_{\Delta \phi}$, plotted as a function of $H_{T}$ in different $y^{*}$ regions (columns) and for different values of $\Delta \phi_{\max }$ (rows), for the LHC and Tevatron scenarios.

$R_{\Delta \phi}$ results on different levels, as

$$
c_{\text {npert }}=c_{\text {hadr }} \cdot c_{\text {ue }} \quad \text { with } \quad c_{\text {hadr }}=\frac{R_{\Delta \phi}^{(2)}}{R_{\Delta \phi}^{(1)}} \quad \text { and } \quad c_{\mathrm{ue}}=\frac{R_{\Delta \phi}^{(3)}}{R_{\Delta \phi}^{(2)}} .
$$

Figure 7 shows the hadronization corrections for $R_{\Delta \phi}$ for the LHC and the Tevatron scenarios. The hadronization corrections for the LHC are very small and always below $1.5 \%\left(0.985<c_{\text {hadr }}<1.00\right)$ at all $H_{T}, y^{*}$, and for all $\Delta \phi_{\max }$ requirements. The hadronization corrections for the Tevatron, although slightly larger, are still always below $6 \%\left(0.94<c_{\text {hadr }}<1.01\right)$. The HERWIG results and the PYTHIA results for the different tunes agree always within $1 \%(3 \%)$, for the LHC (Tevatron) scenario. 

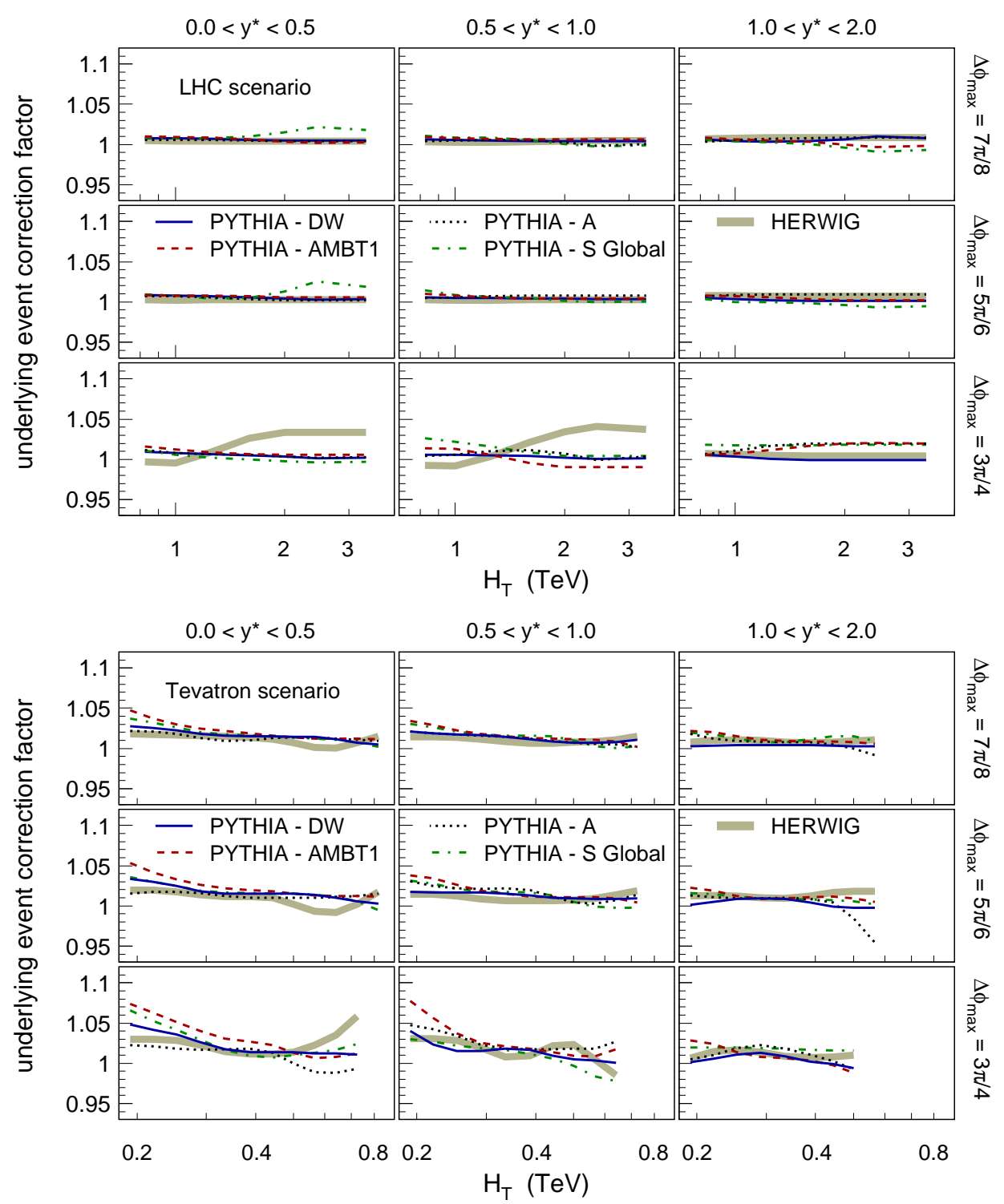

Figure 8. The underlying event corrections for $R_{\Delta \phi}$, plotted as a function of $H_{T}$ in different $y^{*}$ regions (columns) and for different values of $\Delta \phi_{\max }$ (rows), for the LHC and Tevatron scenarios.

The corrections for $R_{\Delta \phi}$ due to effects from the underlying event are displayed in figure 8 for the LHC and the Tevatron scenarios. For the LHC, the underlying event corrections are always below $4 \%\left(0.99<c_{\mathrm{ue}}<1.04\right)$, at all $H_{T}, y^{*}$, and for all $\Delta \phi_{\max }$ requirements. The maximum corrections increase with decreasing $\Delta \phi_{\max }$. They are $2 \%$ for $\Delta \phi_{\max }=7 \pi / 8$, $3 \%$ for $\Delta \phi_{\max }=5 \pi / 6$, and $4 \%$ for $\Delta \phi_{\max }=3 \pi / 4$. For the Tevatron, the underlying event corrections $\left(0.99<c_{\mathrm{ue}}<1.08\right)$ have the same qualitative behavior and they are approximately twice as large as those for the LHC. The HERWIG results and the PYTHIA results for the different tunes are in good agreement. The different model predictions for the LHC (Tevatron) scenario agree better than $2 \%(3 \%)$ for $\Delta \phi_{\max }=7 \pi / 8$, and always better than $4 \%(6 \%)$. 
The total non-perturbative corrections are always in the range $0.98<c_{\text {npert }}<1.03$ $\left(0.96<c_{\text {npert }}<1.03\right)$ for the LHC (Tevatron) scenario. The smallness of these corrections and their small model dependence are remarkable features of the quantity $R_{\Delta \phi}$ which makes $R_{\Delta \phi}$ well-suited for precision tests of pQCD.

\section{Phenomenology}

In this section, we discuss two examples of the potential impact of future $R_{\Delta \phi}$ measurements for QCD phenomenology.

\subsection{Sensitivity to $\alpha_{s}$}

In pQCD, $R_{\Delta \phi}$ is computed as a ratio of three-jet and dijet cross sections, which is, at LO, proportional to $\alpha_{s}$. In the following, we study the sensitivity of $R_{\Delta \phi}$ to $\alpha_{s}$ and investigate the effects of theoretical and experimental uncertainties on the $\alpha_{s}$ results. These studies are made in the kinematic region of $y^{*}<0.5$ and for $\Delta \phi_{\max }=7 \pi / 8$ where the pQCD predictions are most reliable (see section 3.1).

In all studies, $\alpha_{s}$ is varied consistently in the pQCD matrix elements and in the PDF sets. The continuous dependence of the NLO pQCD predictions for $R_{\Delta \phi}$ on $\alpha_{s}$ is obtained using cubic interpolation between the discrete $\alpha_{s}\left(M_{Z}\right)$ values for which the MSTW2008NLO PDFs sets are available. ${ }^{4}$ Where needed, $\alpha_{s}\left(M_{Z}\right)$ is converted from the scale $\mu_{R}=M_{Z}$ to the scale $\mu_{R}=H_{T} / 2$, using the two-loop solution of the renormalization group equation.

A first impression of the $\alpha_{s}$ sensitivity is obtained by studying the $\alpha_{s}\left(M_{Z}\right)$ dependence of the NLO pQCD predictions for $R_{\Delta \phi}$. For this purpose, we plot $R_{\Delta \phi}$ for values of $\alpha_{s}\left(M_{Z}\right)=0.110-0.130$ (labeled $R_{\Delta \phi}\left(\alpha_{s}\left(M_{Z}\right)\right)$ ), normalized to the value of $R_{\Delta \phi}$ for $\alpha_{s}\left(M_{Z}\right)=0.1184$ (the world average value [1]) as a function of $\alpha_{s}\left(M_{Z}\right)$. The results are shown in figure 9 for three different $H_{T}$ bins, for the LHC and the Tevatron scenarios, and are compared to the naive expectation of a linear relation $\left(R_{\Delta \phi} \propto \alpha_{s}\right)$. Deviations from a linear dependence could be due to three different effects.

1. The naive expectation of a linear dependence stems from the LO picture, and is modified due to NLO corrections.

2. The naive expectation assumes a perfect cancellation of the PDFs, while residual PDF effects may lead to non-linearities.

3. While figure 9 shows the $\alpha_{s}\left(M_{Z}\right)$ dependence, the calculations for $R_{\Delta \phi}$ are made for the scale $\mu_{R}=H_{T} / 2$, and the relation between $\alpha_{s}\left(\mu_{R}\right)$ and $\alpha_{s}\left(M_{Z}\right)$ as a function of $\alpha_{s}\left(M_{Z}\right)$ is not linear, and involving logarithms of $\left(\mu_{R} / M_{Z}\right)$.

For $\alpha_{s}\left(M_{Z}\right) \lesssim 0.125$, in the Tevatron scenario, the $\alpha_{s}\left(M_{Z}\right)$ dependence of $R_{\Delta \phi}$ is almost linear for $180<H_{T}<205 \mathrm{GeV}$ (i.e. where $\mu_{R}=H_{T} / 2 \approx M_{Z}$ ). The decrease of the slope (and therefore the increasing non-linearity) of the curves for higher $H_{T}$ is likely caused by the third effect. The change of the slopes around $\alpha_{s}\left(M_{Z}\right) \approx 0.125$ is likely caused by the

\footnotetext{
${ }^{4}$ The MSTW2008NLO PDF sets are available for $\alpha_{s}\left(M_{Z}\right)=0.110,0.111,0.112, \cdots, 0.130$.
} 

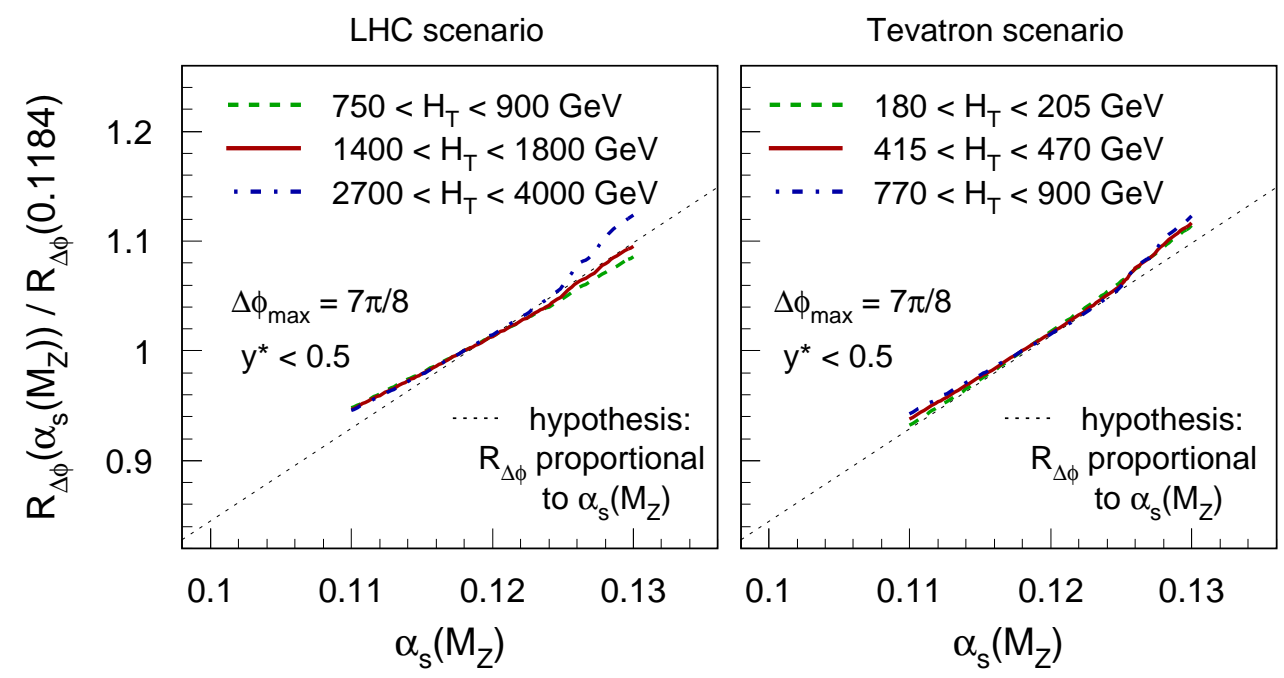

Figure 9. The $\alpha_{s}\left(M_{Z}\right)$ dependence of $R_{\Delta \phi}$, normalized by the $R_{\Delta \phi}$ value at $\alpha_{s}\left(M_{Z}\right)=0.1184$, for the LHC scenario (left) and the Tevatron scenario (right) in three different $H_{T}$ bins for $\Delta \phi_{\max }=$ $7 \pi / 8$ and $y^{*}<0.5$.

second effect. The reduced slope towards high $H_{T}$ implies a slightly reduced sensitivity to $\alpha_{s}\left(M_{Z}\right)$ at the LHC.

Currently, the precision of $\alpha_{s}$ results obtained from hadron colliders which are based on NLO calculations, is limited by theory uncertainties stemming from the renormalization and factorization scale dependencies of the calculations. ${ }^{5}$ Therefore we estimate the corresponding uncertainties for $\alpha_{s}$ extractions from $R_{\Delta \phi}$. In the typical procedure of most $\alpha_{s}$ analyses, the central $\alpha_{s}$ results are derived for a fixed choice of the renormalization and factorization scales. The uncertainties of $\alpha_{s}$ due to the scale dependence are then obtained by repeating the $\alpha_{s}$ fits for variations of the scales around their central values. In the absence of actual $R_{\Delta \phi}$ data, we estimate the corresponding uncertainties for $\alpha_{s}$ by computing the variations in $\alpha_{s}$ which are required to bring the NLO pQCD results at a different scale into agreement with those at the central scale. As discussed in section 3.1, we use a central scale of $\mu_{R}=\mu_{F}=\mu_{0}=H_{T} / 2$ and a range of variations in which $\mu_{R}$ and $\mu_{F}$ are varied independently between $\mu_{0} / 2$ and $2 \mu_{0}$, while never exceeding $0.5 \leq \mu_{R} / \mu_{F} \leq 2.0$. The largest effects of all variations are quoted as the corresponding uncertainties for $\alpha_{s}$. The expected uncertainties for $\alpha_{s}\left(\mu_{R}=H_{T} / 2\right)$, derived using this procedure are shown in figure 10 (left) in the kinematic range $y^{*}<0.5$ and $\Delta \phi_{\max }=7 \pi / 8$, as a function of $\mu_{R}$, for the LHC and the Tevatron. For the LHC scenario, the uncertainties are between $-3 \%$ and $+4 \%$ at high $H_{T}$, and slightly lower at low $H_{T}$. For the Tevatron, the uncertainties are between $-4 \%$ and $+6 \%$. This is a good theoretical precision for testing the running of $\alpha_{s}$ at highest energies.

\footnotetext{
${ }^{5}$ The presently most precise $\alpha_{s}$ result from a hadron collider was obtained using theory calculations beyond NLO (adding the 2-loop corrections from threshold corrections) and has therefore smaller scale uncertainties [34]. These contributions are, however, only available for inclusive jet production and neither for dijet nor for three-jet production.
} 


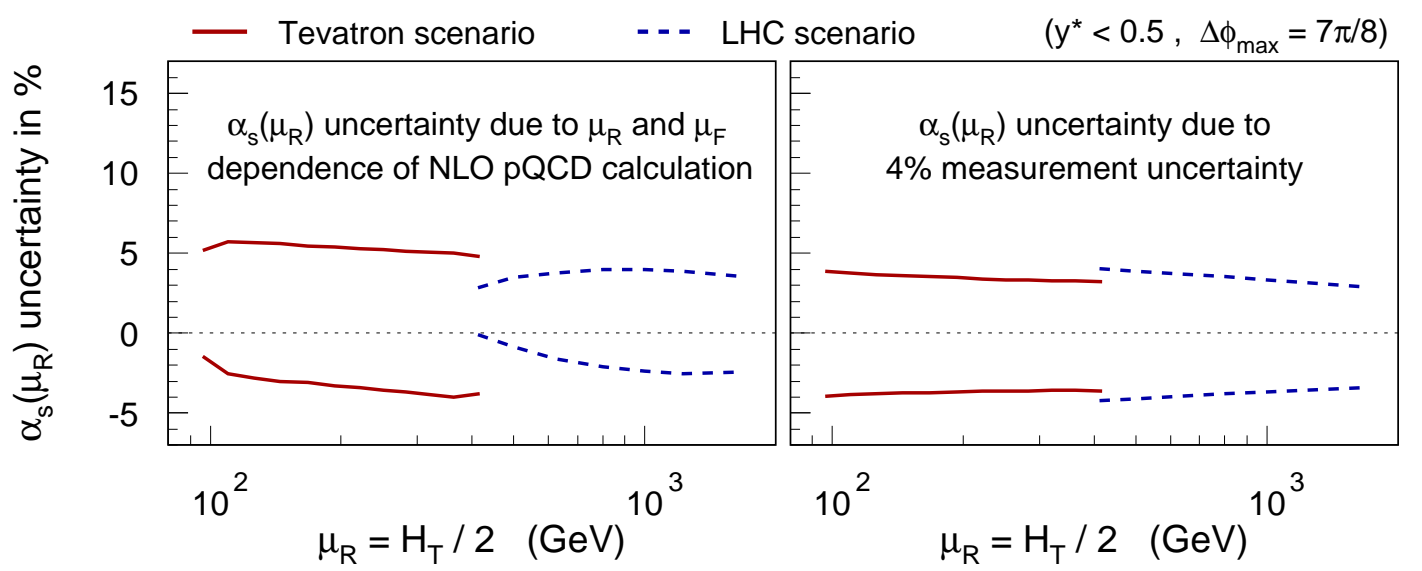

Figure 10. The estimated uncertainties of $\alpha_{s}$ results extracted from $R_{\Delta \phi}$ at the LHC and the Tevatron, at a scale $\mu_{R}=H_{T} / 2$, due to the renormalization and factorization scale dependence of the NLO pQCD calculation (left) and due to an experimental uncertainty of $4 \%$, for $\Delta \phi_{\max }=7 \pi / 8$ and $y^{*}<0.5$.

For comparison, we have also computed the uncertainty of $\alpha_{s}\left(H_{T} / 2\right)$ resulting from an experimental uncertainty of $4 \%$. The results in figure 10 (right) show that for the LHC and the Tevatron scenarios this uncertainty is approximately of the same size as the uncertainty due to the scale dependence. In other words, if the total uncertainty for the $\alpha_{s}$ results should not be limited by the experimental precision, the experiments must measure $R_{\Delta \phi}$ with a precision of at least approximately $4 \%$. Given the precision of recently published measurements of the multi-jet cross section ratios $R_{3 / 2}$ [3-5] and $R_{\Delta R}$ [6], this should be achievable.

\subsection{Event Generator Tuning}

We also compute $R_{\Delta \phi}$ predictions using the Monte Carlo event generators HERWIG 6.520 and PYTHIA 6.426. The HERWIG generator is used with default settings, and for PYTHIA we use tune AMBT1 (derived by the ATLAS collaboration using LHC data) and tune DW (which has been tuned to the previous $\mathrm{D} \varnothing$ measurement of dijet azimuthal decorrelations). The results for $\Delta \phi_{\max }=5 \pi / 6$ are shown in figure 11 for the LHC and the Tevatron scenarios. At central rapidities (i.e. low $y^{*}$ ) the predictions of the different generators agree reasonably well with each other, and also with the NLO pQCD results. The different generators (and tunes), however, predict very different $y^{*}$ dependencies. In the forward region $\left(1<y^{*}<2\right)$, the predictions differ strongly in magnitude and in shape. The HERWIG predictions are more than $30 \%$ higher, and the PYTHIA tune AMBT1 predictions have a very different shape as compared to the NLO pQCD results. The large range of the predictions from different PYTHIA tunes and their differences to HERWIG indicates that measurements of $R_{\Delta \phi}$ at the LHC and at the Tevatron will have strong impact on the future tuning of event generators. 

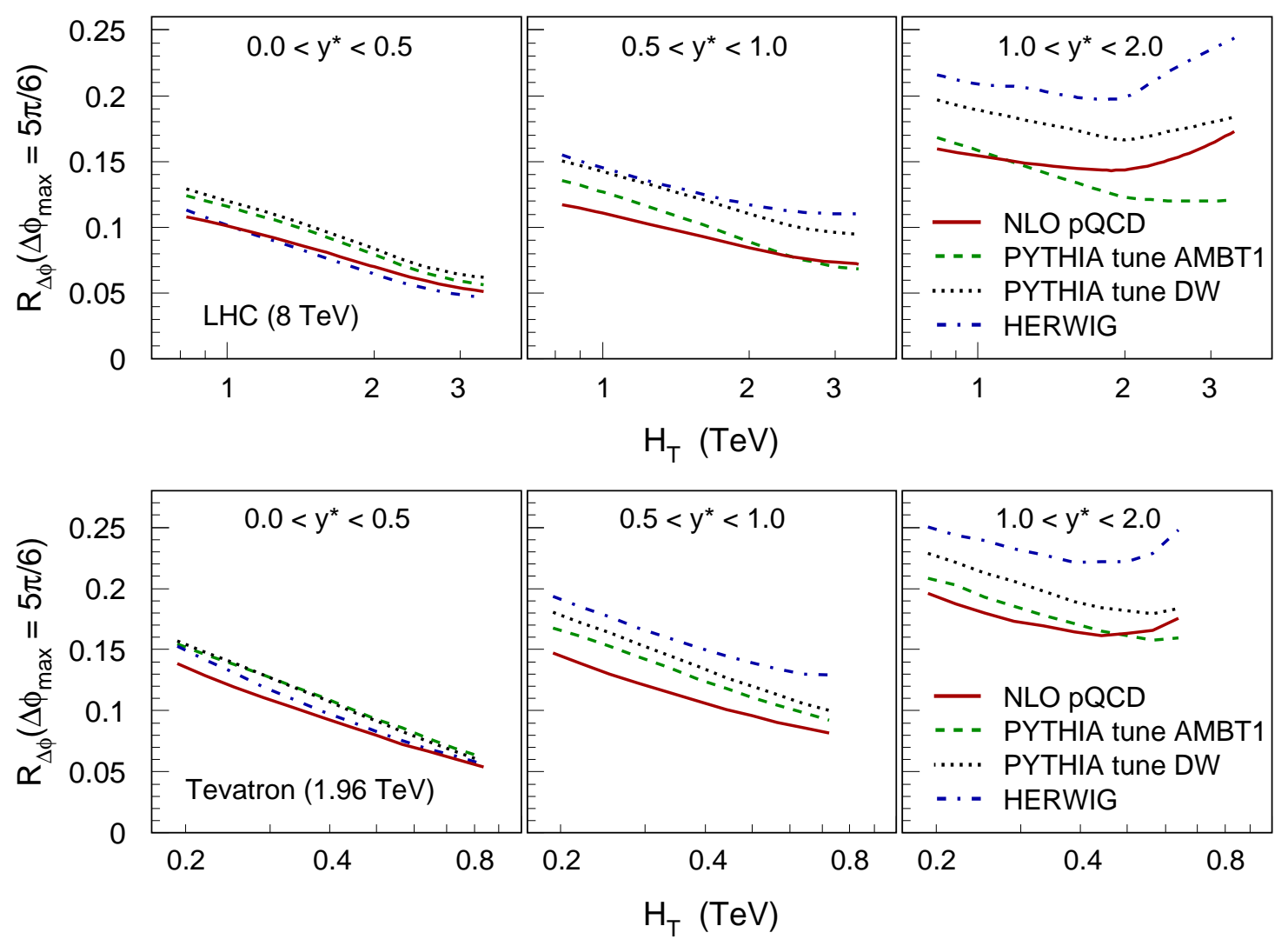

Figure 11. Comparison of $R_{\Delta \phi}$ predictions for $\Delta \phi_{\max }=5 \pi / 6$ of different event generators with the NLO pQCD predictions as a function of $H_{T}$ in different $y^{*}$ regions for the LHC and Tevatron scenarios.

\section{Summary}

We have introduced a new quantity $R_{\Delta \phi}$ for studies of the rapidity and transverse momentum dependence of dijet azimuthal decorrelations at hadron colliders. Since $R_{\Delta \phi}$ is defined as a ratio of cross sections, experimental and theoretical uncertainties, which are correlated between the numerator and the denominator, will cancel to a large extent. We suggest to measure the rapidity and transverse momentum dependence of $R_{\Delta \phi}$ using the longitudinally boost invariant variables $y^{*}$ and $H_{T}$. In pQCD, $R_{\Delta \phi}$ is given by a ratio of three-jet and dijet cross sections, and, at leading order, proportional to $\alpha_{s}$. We have proposed scenarios for measuring $R_{\Delta \phi}$ at the LHC and at the Tevatron, for which we have computed the NLO pQCD predictions and the size of non-perturbative corrections. The non-perturbative corrections are below $3 \%$ with a model dependence of typically less than $2 \%$. The NLO pQCD predictions have PDF uncertainties of less than $1 \%$, and a scale dependence of $4-6 \%$ (for $\Delta \phi_{\max }=7 \pi / 8$ and $\left.5 \pi / 6\right)$. These properties make $R_{\Delta \phi}$, and its $H_{T}$ dependence an ideal quantity for determinations of $\alpha_{s}$ and for studying its running up to the energy frontier.

While these studies have focused on the $H_{T}$ dependence of $R_{\Delta \phi}$, we have also investigated the theoretical predictions for the rapidity dependence. We have shown that, at fixed 
$H_{T}$, NLO pQCD and the event generators PYTHIA and HERWIG all predict an increase of $R_{\Delta \phi}$ with $y^{*}$, however, the rate of the increase differs strongly between the different models. Measurements of $R_{\Delta \phi}$ at the LHC an the Tevatron will be able to distinguish between the different predictions, test NLO pQCD and play an important role in future tuning of Monte Carlo event generators.

\section{Acknowledgments}

We are thankful to Don Lincoln for numerous discussions and for comments on the manuscript. This work has been supported by grants DE-FG02-99ER41117 and DE-FG02-10ER46723 from the U.S. Department of Energy.

\section{References}

[1] J. Beringer et al. (Particle Data Group), The Review of Particle Physics, Phys. Rev. D 86 (2012) 010001.

[2] D0 Collaboration, V. M. Abazov et al., Measurement of three-jet differential cross sections $d \sigma_{3 j e t} / d M_{3 j e t}$ in pp collisions at $\sqrt{s}=1.96 \mathrm{TeV}$, Phys. Lett. B $\mathbf{7 0 4}$ (2011) 434 [arXiv:1104.1986].

[3] CMS Collaboration, S. Chatrchyan et al., Measurement of the Ratio of the 3-jet to 2-jet Cross Sections in pp Collisions at $\sqrt{s}=7$ TeV, Phys. Lett. B 702 (2011) 336 [arXiv:1106.0647].

[4] ATLAS Collaboration, G. Aad et al., Measurement of multi-jet cross sections in proton-proton collisions at a $7 \mathrm{TeV}$ center-of-mass energy, Eur. Phys. J. C 71, 1763 (2011) [arXiv:1107.2092].

[5] D0 Collaboration, V. M. Abazov et al., Measurement of the ratio of three-jet to two-jet cross sections in p $\bar{p}$ collisions at $\sqrt{s}=1.96 \mathrm{TeV}$, [arXiv:1209.1140].

[6] D0 Collaboration, V. M. Abazov et al., Measurement of angular correlations of jets at sqrt(s) $=1.96 \mathrm{TeV}$ and determination of the strong coupling at high momentum transfers, Phys. Lett. B 718 (2012) 56 [arXiv:1207.4957].

[7] D0 Collaboration, V. M. Abazov et al., Measurement of dijet azimuthal decorrelations at central rapidities in p $\bar{p}$ collisions at $\sqrt{s}=1.96$ TeV, Phys. Rev. Lett. 94 (2005) 221801 [hep-ex/0409040].

[8] M. Begel, M. Wobisch, and M. Zielinski, Dijet Azimuthal Decorrelations and Monte Carlo Tuning, in Tevatron-for-LHC Report of the QCD Working Group, FERMILAB-CONF-06-359, [hep-ph/0610012].

[9] CMS Collaboration, V. Khachatryan et al., Dijet Azimuthal Decorrelations in pp Collisions at $\sqrt{s}=7$ TeV, Phys. Rev. Lett. 106 (2011) 122003 [arXiv:1101.5029].

[10] ATLAS Collaboration, G. Aad et al., Measurement of Dijet Azimuthal Decorrelations in pp Collisions at $\sqrt{s}=7$ TeV, Phys. Rev. Lett. 106 (2011) 172002 [arXiv:1102.2696].

[11] R. Dhullipudi, The Study of $p_{T}$ Dependence of Dijet Azimuthal Decorrelations in Proton-Proton Collisions at $\sqrt{s}=7 \mathrm{TeV}$, Ph.D. dissertation, Louisiana Tech University (2012). 
[12] K. Chakravarthula, Study of Jet Transverse Momentum and Jet Rapidity Dependence of Dijet Azimuthal Decorrelations with the Do Detector, Ph.D. dissertation, Louisiana Tech University (2012).

[13] M. Cacciari, G. P. Salam, and G. Soyez, The Anti-k(t) jet clustering algorithm, JHEP 0804 (2008) 063 [arXiv:0802.1189].

[14] G. C. Blazey et al., Run II Jet Physics, in: U. Baur, R. K. Ellis, and D. Zeppenfeld (Eds.), Proceedings of the Workshop: QCD and Weak Boson Physics in Run II, Fermilab-Pub-00/297 (2000).

[15] M. Cacciari, G. P. Salam, and G. Soyez, FastJet User Manual, Eur. Phys. J. C 72 (2012) 1896 [arXiv:1111.6097].

[16] M. Cacciari and G. P. Salam, Dispelling the $N^{3}$ myth for the $k_{t}$ jet-finder, Phys. Lett. B 641 (2006) 57 [hep-ph/0512210].

[17] Z. Nagy, Next-to-leading order calculation of three jet observables in hadron hadron collision, Phys. Rev. D 68 (2003) 094002 [hep-ph/0307268].

[18] Z. Nagy, Three jet cross-sections in hadron hadron collisions at next-to-leading order, Phys. Rev. Lett. 88 (2002) 122003 [hep-ph/0110315].

[19] T. Kluge, K. Rabbertz, and M. Wobisch, FastNLO: Fast pQCD calculations for PDF fits, in M. Kuze, K. Nagano, K. Tokushuku, K. Hackensack (Eds.), Proceedings of the XIV Workshop on Deep Inelastic Scattering, [hep-ph/0609285].

[20] W. A. Bardeen et al., Deep Inelastic Scattering Beyond The Leading Order In Asymptotically Free Gauge Theories, Phys. Rev. D 18 (1978) 3998.

[21] A. D. Martin et al., Parton distributions for the LHC, Eur. Phys. J. C 63 (2009) 189 [arXiv:0901.0002].

[22] H. L. Lai et al., New parton distributions for collider physics, Phys. Rev. D 82 (2010) 074024 [arXiv:1007.2241].

[23] R. D. Ball et al., Impact of Heavy Quark Masses on Parton Distributions and LHC Phenomenology, Nucl. Phys. B 849 (2011) 296 [arXiv:1101.1300].

[24] G. Corcella et al., HERWIG 6: An Event generator for hadron emission reactions with interfering gluons (including supersymmetric processes), JHEP 0101 (2001) 010 [hep-ph/0011363].

[25] G. Corcella et al., HERWIG 6.5 release note, [hep-ph/0210213].

[26] T. Sjöstrand et al., High-energy physics event generation with PYTHIA 6.1, Comput. Phys. Commun. 135 (2001) 238 [hep-ph/0010017].

[27] T. Sjöstrand, S. Mrenna, and P. Z. Skands, PYTHIA 6.4 Physics and Manual, JHEP 0605 (2006) 026 [hep-ph/0603175].

[28] R. Field, Tevatron Run 2 Monte-Carlo Tunes in Tevatron-for-LHC Report of the QCD Working Group, FERMILAB-CONF-06-359, [hep-ph/0610012].

[29] R. Field, Min-Bias and the Underlying Event at the Tevatron and the LHC, talk presented at the Fermilab ME/MC Tuning Workshop, Fermilab, October 4, 2002.

[30] G. Brandt, Charged particle multiplicities in inelastic $p$ p interactions with ATLAS, in: M. Diehl, J. Haller, T. Schörner-Sadenius, and G. Steinbrück (Eds.), 5th Conference: Physics at the LHC 2010, DESY-PROC-2010-01 (2010). 
[31] H. Schulz and P. Z. Skands, Energy Scaling of Minimum-Bias Tunes, Eur. Phys. J. C 71 (2011) 1644 [arXiv:1103.3649].

[32] T. Sjöstrand and P. Z. Skands, Multiple interactions and the structure of beam remnants, JHEP 0403 (2004) 053 [hep-ph/0402078].

[33] T. Sjöstrand and P. Z. Skands, Transverse-momentum-ordered showers and interleaved multiple interactions, Eur. Phys. J. C 39 (2005) 129 [hep-ph/0408302].

[34] D0 Collaboration, V. M. Abazov et al., Determination of the strong coupling constant from

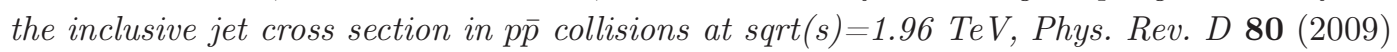
111107 [arXiv:0911.2710]. 